\title{
La rhétorique de la qualification et les controverses d'étiquetage
}

The Rhetoric of Qualification: Labelling Controversies

\section{Marc Angenot}

\section{(2) OpenEdition \\ 1 Journals}

Electronic version

URL: http://journals.openedition.org/aad/1787

DOI: 10.4000/aad.1787

ISSN: 1565-8961

Publisher

Université de Tel-Aviv

\section{Electronic reference}

Marc Angenot, "La rhétorique de la qualification et les controverses d'étiquetage », Argumentation et Analyse du Discours [Online], 13 | 2014, Online since 14 October 2014, connection on 23 September 2019. URL : http://journals.openedition.org/aad/1787 ; DOI : 10.4000/aad.1787

This text was automatically generated on 23 September 2019.

\section{(c) $(1) \odot$}

Argumentation \& analyse du discours est mis à disposition selon les termes de la licence Creative Commons Attribution - Pas d'Utilisation Commerciale - Pas de Modification 4.0 International. 


\title{
La rhétorique de la qualification et les controverses d'étiquetage
}

\author{
The Rhetoric of Qualification: Labelling Controversies
}

Marc Angenot

1 Que ce soit dans le domaine des sciences, dans celui de la philosophie, dans les institutions académiques, les sciences sociales et historiques, les mondes artistique et littéraire, partout et constamment, éclatent des polémiques et se déclenchent des controverses intellectuelles souvent véhémentes qui, très souvent aussi, quittent l'empyrée des spécialistes pour aboutir sur la scène publique et devenir de véritables événements sociaux et politiques. Or, il fallait constater jusqu'à tout récemment l'absence en langue française de travaux de problématisation, de discussion des enjeux, de théorisation de cette histoire des controverses intellectuelles et des polémiques publiques. L'Apologie de la polémique de Ruth Amossy est venue heureusement combler en partie cette lacune. Néanmoins, l'histoire des grandes controverses demeure limitée à quelques bons échantillons en français. Elle forme au contraire un secteur de l'histoire intellectuelle particulièrement bien développé et académiquement identifié en domaine allemand. Il a fallu du reste que ce soit un chercheur suisse alémanique, Jürg Altwegg qui esquisse avec érudition une histoire des polémiques intellectuelles en France du $18^{\mathrm{e}}$ siècle à 1989 dans son ouvrage Republik des Geistes ${ }^{1}$. Il existe sans nul doute de nombreux livres et pamphlets qui sont parties prenantes de tel et tel débat d'idées, mais l'approche de ces débats en historien des idées fait grandement défaut en domaine français.

Dans un essai d'hommage à Chaïm Perelman, Pierre-André Taguieff a proposé un correctif à la rhétorique consensuelle qui est celle du philosophe bruxellois en mettant de l'avant un aspect inhérent, à son sentiment, à tout échange rhétorique, "les éléments de ce que j'appellerai, dit-il, une polémologie discursive " (Taguieff 1990 : 261). Taguieff retient la polémicité parmi les traits par lesquels l'argumentation rhétorique se distingue de la démonstration logique. C'est un amendement essentiel. Je n'ai cessé de travailler dans ce sens et ce n'est pas par hasard que ce sont des historiens des idées qui se rencontrent pour avancer ce critère : toute histoire des idées politiques 
fait apparaître continûment des affrontements de thèses irréconciliables, des dialogues de sourds, des controverses interminables entre des positions défendues bec et ongles.

Or, il se fait que les dites controverses savantes ou publiques, résurgentes et irréconciliables, ne portent souvent que sur la qualification - au sens juridique de ce terme - d'un fait, d'un acte, d'un événement ou d'un individu, d'une entité politique. C'est ce cas particulier, cas très répandu en réalité, qui fera l'objet de ces quelques pages. Les controverses les plus âpres et interminables (ne) portent souvent (que) sur les mots avec lesquels on classe et on désigne et l'âpre débat sur les catégories et les dénominations démarre au quart de tour. Ces «querelles de mots » avec leurs enjeux souvent peu compréhensibles à qui n'est pas « dans le coup ", sont de très grand poids et la défense par chacun de son propre vocabulaire et de ses propres classements semble ressentie comme vitale. La " qualification juridique » est l'opération qui permet l'application d'une règle de droit en appréhendant des éléments de fait et en inscrivant ceux-ci dans une catégorie du droit positif en recourant notamment à des précédents jurisprudentiels. L'attribution d'une qualification juridique permet à son tour l'application du régime juridique correspondant, c'est-à-dire l'ensemble de règles de droits applicables à une personne, une organisation, une activité, etc. Je propose d'étendre ce concept au-delà du monde des prétoires pour l'appliquer à un phénomène diffus de la vie intellectuelle et de la vie en société: les polémiques les plus insurmontables n'ont souvent pas d'autre objet que la prétention de classer de telle ou telle façon et dénommer sans qu'il y ait apparemment d'autre enjeu concret et pratique.

4 Raisonner et argumenter, c'est, au plus élémentaire de ses opérations, distinguer, identifier et classer. Tout commence en rhétorique, dans le discours social et dans les idéologies, en donnant des noms aux choses, en faisant par exemple avec deux mots, deux entités distinctes de ce qui, selon d'autres, est indissociable, de ce qui forme les deux faces ou les étapes d'un seul processus. Ou au contraire en assimilant sous un seul terme ce que " tout » devrait distinguer. Or, rien, ni dans le langage ni dans le rapport de l'intelligence au monde, n'interdit indubitablement de créer des entités hétérogènes, des distinctions déraisonnables et des amalgames fallacieux.

Bien des polémiques persistantes et amères ne tiennent - du moins techniquement qu'à une seule définition divergente : un embryon est-il une "personne humaine »? À coup sûr, si votre définition de la « personne humaine » est faite pour englober le foetus dès la conception. On ne peut en effet pas réfuter une définition mais simplement lui en opposer une autre.

6 Parmi les voies méthodologiques originales ouvertes dans les sciences humaines dans l'Allemagne contemporaine, on peut mentionner les travaux de Reinhart Koselleck et de ses disciples qui ont développé une problématique dénommée Begriffsgeschichte, l'histoire conceptuelle ou sémantique historique ${ }^{2}$. Or cette histoire est indissociablement une histoire polémique, une histoire de polémiques. Les Grundbegriffe, les catégories fondamentales d'une culture politique donnée n'évoluent que dans des controverses successives opposant classements et définitions contradictoires.

7 Je me borne dans les pages qui suivent à circonscrire et illustrer sommairement ce que je vois comme un vaste domaine d'enquête, à signaler des problématiques prometteuses et esquisser une approche et une méthode. 


\section{Trois exemples}

8 Trois exemples, datés de la même journée, récente, illustrent la fréquence du phénomène et l'intensité des réactions qui polarisent ordinairement la sphère publique en deux «camps » armés de ses arguments unilatéraux, - et qui séparent aussi du même coup dans les champs disciplinaires, les doctes et les experts :

9 - Santiago du Chili (AFP), 4 octobre 2013 : le Conseil chilien de l'éducation nationale, répondant à la demande de "clarification" de la ministre de l'éducation, Carolina Schmidt, recommande, au bout de plusieurs années de discussion politiquement chargées et étrangères à de "pures " considérations pédagogiques, que "dictature " soit substitué dans les manuels de lycée aux mots de "régime militaire " retenus jusque-là pour qualifier le régime dirigé par le général Pinochet entre 1973 et 1990.

10 - Paris, Le Monde, 4 octobre 2013 : Marine Le Pen, désireuse d'améliorer l'image de son parti, le Front national, censément devenu fréquentable, récuse l'étiquette d'« extrême droite » accolée à ce parti créé par son père en 1972 et qu'elle dirige depuis trois ans. "Il s'agit d'un terme volontairement péjoratif», proteste-t-elle, d'une "injure», utilisée pour "nuire » en mettant le FN « dans le même sac " que le mouvement néonazi grec Aube dorée. Elle envisage de saisir la justice pour faire cesser ce qu'elle considère comme une "insulte» et une " "guerre sémantique" contre son mouvement ». À la guerre comme à la guerre : Le Monde réplique en titrant impavide son éditorial du lendemain : « Le Front national, parti d'extrême-droite ». « Redisons-le donc clairement, réaffirme l'éditorial: par sa seule position sur l'échiquier politique français, autant que par ses idées et son projet, le Front national est, aujourd'hui comme hier, un mouvement d'extrême droite ». L'appel aux experts vient en renfort: «Je considère, comme l'immense majorité de mes collègues, que le FN est d'extrême droite, par son histoire et par son positionnement objectif dans le champ politique ", explique le chercheur Joël Gombin à l'AFP3. Un député socialiste, Thierry Mandon renchérit sur l'insulte en inventant une catégorie hyperbolique de son crû : le FN est « un parti national-fasciste $»^{4}$. Cette polémique se déclenche au moment où Marine Le Pen bénéficie d'enquêtes d'opinion montrant une adhésion marquée d'une partie de l'électorat à ses idées. Apparemment, la qualification que la leader du Front national serait prête à admettre, c'est « droite nationale ».

11 Dans le cas du Front national, la querelle de catégorisation dure depuis quarante ans: c'est une double controverse, à la fois publique et savante, car les politologues se disputent non moins entre eux, alors même qu'ils récusent les étiquetages purement dénonciateurs et injurieux de la polémique publique. Dans la vie publique, le Front national concentre depuis quarante ans les haines, les fantasmes aussi, et incarne à gauche la menace fasciste résurgente, croissante, jamais vaincue. Du côté des doctes toutefois, Pierre-André Taguieff écarte le catégorème " [néo-]fasciste », devenu vide de sens, tandis qu'il considère "extrême droite" non moins flou et trop polémique. Taguieff retient, faute de mieux, "populisme", catégorie dont nul ne se réclame comme telle, car nul ne reconnaît jamais ni ne proclame, que ce soit à droite ou à gauche, "Je suis un populiste!» Mais cette catégorie qui, dans la sphère publique, fonctionne comme un étiquetage péjoratif à tout-va, permet précisément à Taguieff et quelques autres, en tant que politologues voués à la sérénité objective, d'écarter les encore plus péjoratifs et vides "fasciste » et «extrême droite ». On rencontre ainsi deux conflits de qualification, l'un savant, l'autre populaire, à la fois indépendants et 
qui pourtant se touchent. "National-populisme", spécifie finalement Pierre-André Taguieff pour classer et étiqueter le F. N. en évitant de le « diaboliser » sommairement mais que veut-il dire en conservant, de la phraséologie à laquelle il s'efforce de conférer un sens serein et objectif, cette antéposition de «national-»qui relève des langues germaniques?

12 - Troisième occurrence, le même jour, d'une qualification quasi-juridique qui déclenche la controverse et des contre-qualifications stridentes : le Conseil de l'Europe estime que la circoncision des garçons pour motifs religieux constitue une «violation de l'intégrité physique » d'un enfant. Réactions indignées de la presse d'Israël qui parle de «racisme» et du Conseil français du culte musulman qui évoque non moins routinièrement l'« islamophobie ».

13 La presse française de ce jour-là, unanime par exception, qualifie de "néo-nazis » les militants grecs du parti Aube dorée. Le Figaro titre le 4 octobre 2013 : "Grèce : le chef des néonazis d'Aube dorée en détention provisoire ». Mais en Grèce, le parti de l'Alliance populaire, $\Lambda \alpha$ «о $\Sigma \cup v \delta \varepsilon \sigma \mu о \varsigma$, en revanche, - irrédentiste, xénophobe, antiimmigrés, antisémite, - ne s'est jamais défini tel et rejette expressément cette appellation!

\section{L'emprunt par l'historien de catégories juridiques}

14 Le droit et l'historiographie partagent des paradigmes fondamentaux, ceux de la recherche de la vérité sur des faits du passé, ceux de l'enquête, de la présentation de pièces à conviction, du témoignage et de son évaluation (selon la sorte de jurisprudence que forme la «critique historique »), des "preuves » enfin, - s'il est aussi vrai que les principes juridiques ne peuvent pas être transférés tels quels dans la recherche historique, que les exigences en matière de preuve ne sont pas de même nature et que différence décisive, mais pas toujours observée, il s'en faut, - l'historien n'est pas censé, au bout de sa reconstitution des faits, si « incriminants » soient-ils, passer jugement ni formuler un réquisitoire (ni un plaidoyer d'acquittement) contre les hommes du passé.

15 - La Kolyma fut-elle un réseau de " camps » au sens où Buchenwald (ou Treblinka, ce qui fait deux) furent des «camps»? Les génocides des Tasmaniens, des Sioux et des Indiens des plaines, des Arméniens, des Juifs, des Tsiganes, des Ukrainiens, des Tutsis sont-ils au même titre définitionnel des "génocides " ${ }^{5}$ ? Question certes juridique d'apparence, mais qui est aussi âprement débattue par les historiens et par les politologues. Le massacre des koulaks par les bolcheviks fut-il un "génocide de classe " (Ernst Nolte dixit)? La même essence en dépit d'accidents différents, pour parler comme les aristotéliciens ? Ou bien ce syntagme de " génocide de classe » est-il absurde à sa face même? Questions juridiques brûlantes non moins qu'historiographiques et qu'éminemment politiques, accompagnées dès lors de controverses interminables.

\section{« Génocide »}

En abordant la prolifération contemporaine, en dehors des prétoires, des arguments par qualification juridique, leur usage et mésusage, il faut traiter de la question connexe, non moins matière à controverses, de l'extension de la catégorie, extension censée conserver les connotations attachées au cas-type et à l'horreur morale qu'il 
inspire. La qualification juridique relève de l'autorité impersonnelle de la loi, mais cette opération toute technique refoule un pathos, une émotivitéqui tend à resurgir. Je prends donc un exemple éminent et prégnant des controverses de qualifications juridiques utilisées par les historiens, les essayistes, les journalistes, en m'arrêtant précisément à la catégorie de " génocide».

Le mot de génocide est un néologisme hybride, gréco-latin, formé en 1944 par Raphael Lemkin, professeur de droit américain d'origine juive polonaise, à partir de la racine

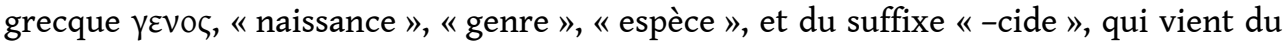
latin caedere, "tuer ». Le terme est apparu pour la première fois dans son étude Axis Rule in Occupied Europe en 1944 (chapitre IX) pour tenter de définir les crimes perpétrés par les nazis à l'encontre des peuples juif, slaves et tsigane durant la Seconde Guerre mondiale, ceux commis par le gouvernement des Jeunes-Turcs de l'Empire ottoman à l'encontre des Arméniens pendant la Première Guerre mondiale, et ceux dont furent victimes les Assyriens en Irak en 1933 (Lemkin 1944). (La persécution des Tsiganes par les nazis n'a pas été abordée durant le Procès de Nuremberg, il est pourtant certain qu'il y avait aussi eu " génocide » dans le cadre juridique alors établi.)

La catégorie juridique est appliquée à Nuremberg en 1945 à la Shoah, puis reprise et appropriée après cette date par les Arméniens, les Ukrainiens, les survivants (quand il s'en trouve) des peuples décimés d'Amérique et d'Australasie, ${ }^{6}$, par les Tutsis - en conservant une charge émotive telle que discuter de la pertinence du qualificatif de "génocide " pour le massacre de Srebrenitsa (le meurtre de 8000 hommes et enfants bosniaques par l'armée de la Republika srpska de Radko Mladičㄱ) serait considéré comme l'amorce d'un négationnisme.

19 Les gouvernements et leurs historiens officiels renâclent à retenir la qualification de génocide pour les massacres commis par les leurs. La Turquie ne nie pas les tueries commises pendant la Grande guerre par le gouvernement Jeune-Turc (M. Erdogan vient de présenter un peu tard ses "condoléances ») mais elle en conteste énormément l'ampleur et rejette la qualification. L'Union Européenne a reconnu le génocide des Arméniens en juillet 1997, mais l'article 312 du Code pénal turc rend passible de cinq ans de prison quiconque évoque publiquement le «prétendu génocide». Orhan Pamuk, Prix Nobel 2006, a fait l'objet de menaces contre sa vie pour avoir affirmé à un journal suisse qu'entre 1915 et 17 « un million d'Arméniens et 30.000 Kurdes ont été tués sur ces terres ». En février 2007, Pamuk, à la suite de menaces faisant suite à l'assassinat de Hrant Dink, s'est installé aux Etats-Unis. L'Assemblée nationale en France a adopté au contraire en octobre 2006 un article de loi pénalisant la négation du génocide arménien.

Par contre, les historiens français n'appliquent pas volontiers à leur passé colonial la catégorie de "génocide» que les historiens ci-devant «indigènes » appliquent notamment au massacre de Sétif de mai 1945 avec ses 40.000 morts et aux massacres commis par l'armée française à Madagascar en 1947 avec peut-être 100.000 victimes.

21 La France a multiplié les lois qui imposent à l'historien de telles qualificationsjuridiques : la Loi Gayssot (1990) promulguée contre les négationnistes de la Shoah a été suivie de la Loi sur le génocide arménien dont je viens de faire état, puis de la Loi Taubira sur le caractère de " crime contre l'humanité » de la traite négrière et de l'esclavage. 

interprétatif, est appliquée par plusieurs historiens à différents épisodes de l'histoire de l'URSS. La question de la qualification juridique de l'Holodomor, la famine de 1932-33 en Union soviétique, centrée sur l'Ukraine a fait l'objet de nombreux travaux, fondés désormais sur archives, depuis l'essai pionnier de Robert Conquest, Harvest of Sorrow en 1986. Le Français Nicolas Werth, après avoir hésité par scrupule de méthode, accepte désormais la qualification de génocide, un " génocide par la faim ", pour l'Holodomor qui fit, selon les estimations atrocement contradictoires des historiens, entre 2,61 et 6 millions de victimes ukrainiennes. La famine concomitante au Kazakhstan, entraînée par la politique de "sédentarisation" de ce peuple nomade, a coûté la vie à 1,45 millions de Kazakhs, soit $38 \%$ de la population totale - ce serait le pourcentage le plus élevé de morts pour une nationalité soviétique dans les années staliniennes (Naimark $2010: 76)$. minorités polonaises, turcophones et coréennes de l'URSS, les déportations de masse ultérieures et les massacres des Tatars de Crimée, des Tchétchènes, des Ingouches et autres peuples du Caucase du Nord ${ }^{8}$, des Allemands de la Volga, des Karatchaïs, des Balkars, des Kalmouks et autres "peuples ennemis » sous Staline sont de plus en plus souvent avec un effort de précision d'ordre juridique et de collection et confrontation des faits, qualifiées de " génocides".

Les livres qui concluent à la pertinence de la catégorie de génocide(s) pour l'histoire de l'URSS se multiplient donc de nos jours - ils n'abondent pas en français. Historien et juriste américain, Norman M. Naimark a publié Stalin's Genocides en 2010. Le but de son essai est d'argumenter en droit international «that Stalin's mass killings of the 1930s should be classified as "genocide" ". L'auteur examine successivement la dékoulakisation de 1929-31, la famine ukrainienne et les campagnes meurtrières contre plusieurs « nationalités » qui se succèdent de 1934 à la Guerre. Il inclut bien entendu les épisodes de meurtre de masse de la Grande terreur de 1937-38. Le massacre de Katyn en 1940 doit également se qualifier de génocide selon les historiens et juristes polonais. Sur tous ces sujets, il y a désormais une littérature considérable basée sur les archives $\mathrm{du}$ Kremlin et provenant notamment d'historiens russes et d'autres historiens du cidevant Pacte de Varsovie.

\section{Extension de la qualification. Abus de langage et intimidation}

Comme il arrive constamment en rhétorique éristique, des expressions dérivées et juridiquement de moins en moins fondées se sont mises à proliférer, lesquelles profitent en quelque sorte de l'aura condamnatrice du concept initial. L'expression de « génocide culturel » est employée de façon souvent laxiste pour décrire la destruction intentionnelle du patrimoine culturel d'un peuple. L'arabisation de la vie publique menée par les gouvernements successifs depuis le temps de deux générations au Maroc et en Algérie est ainsi dénoncée sur de nombreuses tribunes internationales par les Amazighs comme un " génocide culturel » anti-berbère.

De « génocide » découlent divers néologismes et calques pseudo-juridiques brandis par plusieurs catégories de victimes alléguées: "ethnocide", qui est le plus répandu 
dénote la destruction de l'identité culturelle d'un groupe, sans détruire physiquement ce groupe. "Linguicide » serait l'acte de «tuer » une langue : le terme est utilisé par Claude Hagège (2001). La France serait par exemple coupable de « linguicide » à l'égard de l'occitan et autres langues vernaculaires. Toutefois, "génocide culturel» peut se trouver étendu à ce " cas » : des nationalistes bretons accusent la France de " génocide culturel de la langue bretonne ", sur le site AlterMedia, 2 septembre 2005. « Démocide " est un terme créé par le politologue R. J. Rummel pour disposer d'un concept plus large que la seule définition légale de génocide. "Ethnocide » est aussi fréquemment utilisé à propos de la destruction par les «Blancs » des cultures des Amérindiens d'Amérique du Nord, - mais le terme est aussi invoqué de façon dénonciatrice pour la sinisation systématique du Tibet par le régime de Pékin.

Brigitte Bardot fait part au monde depuis plusieurs années de son indignation devant le massacre par les chasseurs canadiens des "bébés phoques", c'est son droit, mais la Fondation Brigitte Bardot dénonce sur son site un « génocide » en cours et c'est abuser $\mathrm{du}$ langage d'une façon qui peut choquer, - choquer notamment les victimes et survivants des « vrais " génocides. «La chasse aux phoques : génocide animalier », titre derechef un autre site anti-fourrure.

Quelques autres exemples banaux et répandus à connotation juridique. Depuis quelques années, l'expression « prise d'otage/pris en otage » est mise à toutes les sauces jusqu'à l'insignifiance. "Les éducatrices de garderie font une journée de grève: les parents sont pris en otage. Des zozos bloquent un pont pendant vingt minutes: les automobilistes sont pris en otages $»^{10}$ - à moins que les malheureux usagers ne soient qualifiés par les médias de "victimes collatérales» du conflit. Le Syndicat du Livre français fait une journée de grève le 6 février 2013, les quotidiens parisiens ne paraissent pas ce jour-là : les marchands de journaux dénoncent à hauts cris la «prise d'otage » qui leur est infligée - la formule sera répétée en boucle aux nouvelles de 8 heures. L'abus croissant dans les médias et sur le web de la qualification [pseudo-]juridique stridente et hyperbolique tient évidemment au caractère de plus en plus fugace de l'information où chaque lobby qui voit midi à sa porte pense qu'il lui faut crier de plus en plus fort pour se faire entendre et retenir l'attention un bref instant.

Une violente polémique récente a illustré de façon inquiétante pour la liberté académique soumise à la pression de lobbies tonitruants bien organisés et indifférents au ridicule comme à la bonne foi - la fonction d'intimidation qui s'attache de nos jours à l'usage, imposé aux autres sous peine de poursuites, de qualifications juridiques abusives. Professeur à l'Université de Lorient et historien de l'esclavage, Olivier PétréGrenouilleau est l'auteur de Traites négrières (2005). Il se voit attaqué au civil par un groupe de pression de la France d'outre-mer, le «Collectif DOM » pour « contestation de crime contre l'humanité »: on lui reproche d'avoir relativisé et même nié la nature criminelle de l'esclavage, d'en avoir contesté le caractère de «crime contre l'humanité » dans un entretien publié par le Journal du dimanche du 12 juin $2005^{11}$. PétréGrenouilleau avait déclaré: "Les traites négrières ne sont pas des génocides " expliquant un peu laborieusement que la Traite n'avait pas pour but d'exterminer un peuple, que le but des Occidentaux était de préserver la main-d'œuvre servile, non de l'anéantir ${ }^{12}$. - Cette affaire est typique: un groupe de pression, résolu à faire l'événement, instrumentalise l'appareil législatif pour crier à la persécution et intimider un chercheur qui n'est pas au service inconditionnel de ses revendications. L'Affaire Pétré-Grenouilleau a été pour nombre d'historiens, échaudés par l'abus de 
plus en plus fréquent d'autres "lois mémorielles ", la goutte qui faisait déborder le vase : ceux-ci ont diffusé en décembre 2005 une pétition intitulée Liberté pour l'histoire! qui a reçu le soutien de près de six cents chercheurs ${ }^{13}$. Elle réclame l'abrogation partielle des différentes lois ayant trait à leur domaine de compétence, notamment de la loi Taubira de 2001 qui impose de reconnaitre l'esclavage et la traite comme «crime contre l'humanité » - qualification, qu'elle soit juridique ou morale, qui n'était du reste contestée par aucun des intervenants. Il a fallu qu'un vieil historien modéré qui avait pourtant l'oreille du pouvoir, René Rémond, publie une critique globale de toutes les législations chargées d'imposer la Vérité historique, Quand l'État se mêle de l'histoire et qu'il réclame au nom de la profession historienne leur retrait en bloc (2006).

\section{Étiquetage et diabolisation}

Certains concepts présentent le monde «sous une forme jugée ». L'expression est de Roland Barthes dans Le degré zéro de l'écriture : " totalitarisme ", " néolibéralisme ", etc., d'une certaine manière, avec ces catégorisations, ces étiquettes idéaltypiques tout est dit: si vous acceptez ce vocable pour qualifier une opinion, une théorie, vous vous prédisposez à endosser le blâme et les conclusions qui vont avec. Le procédé de l'étiquetage condamnateur s'attribue motu proprio une autorité, l'apparence au moins d'une délégation d'autorité, du seul fait qu'il qualifie de façon quasi-juridique, que celui qui l'avance se mue en procureur et transforme l'opinion adverse en autre chose qu'une "simple " opinion : une faute, un délit ou même, dans un vocabulaire daté qui garde une prégnance, un blasphème contre le sacré civique et politique, quelque chose enfin qui se désigne à l'indignation publique. Il est des catégorèmes qui sont en euxmêmes toute une argumentation ou plutôt qui en permettent avantageusement l'économie tout en cherchant à intimider. L'étiquetage accusateur et « diabolisant » est en progrès de nos jours à mesure que le règne des médias « chauds » et des soundbites favorise l'outrancier et décourage la peu spectaculaire discussion sereine et pondérée. La liste de base des " étiquettes » se dresse aisément : "fasciste », " raciste », les plus anciennes, à quoi sont venus s'adjoindre successivement "sexiste", "homophobe ", «islamophobe». On observe une montée en puissance de ce sommaire moyen rhétorique de mettre fin au débat en clouant au pilori l'adversaire dont témoigne un néologisme à succès immédiat, "diabolisation » - par anglicisme " démonisation ». "Terrorisme intellectuel ", "police de la pensée ", «nouvelle inquisition » sont les contre-feux et contre-étiquetages indignés des gens visés par les manœuvres diabolisatrices.

\section{«Fasciste ! »}

Je reviens à «fascisme » comme un cas éminent de suremploi et de déperdition de sens - et comme modèle historique d'un phénomène qui s'est emballé. L'antifascisme d'avant la Deuxième Guerre mondiale a légué à la gauche d'après 1945 une catégorie nébuleuse qui allait être en perpétuelle expansion, un « fascisme » sans rivage devenu l'injure suprême semée à tout vent. « En France, on est toujours, ou l'on a été, le fasciste de quelqu'un ", constate-t-on (Machefer 1974, 1er §). Pascal Ory notamment s'agace de cette inflation : à la fin du $20^{\mathrm{e}}$ siècle, ironise-t-il, les gauchistes identifient la démocratie occidentale au "fascisme» et au "nazisme» - et leurs adversaires répliquent en 
qualifiant de «fasciste» ou de «totalitaire» leur rhétorique de la contestation (Ory 1995 : 34). La critique de l'abus à fonction intimidatrice de "fascisme» ne pouvait que déboucher sur le procès d'un antifascisme décrété imposteur, manipulé par les communistes, procès qui a commencé à s'instruire en dépit d'une longue intimidation dans les années 1970. On a assisté depuis 1945, c'est-à-dire depuis la chute des régimes effectivement "fascistes", à une vaine "multiplication de fascismes imaginaires". L'antifascisme tel qu'il s'est dégradé au bout de ces abus d'usage se ramène de nos jours à un misérable « ensemble d'attitudes mentales, de représentations stéréotypées et de croyances sloganisées » que Pierre-André Taguieff montre nocives à la vie civique non moins qu'absurdes (2007: 324, 460). Tout régime répressif est «fasciste» pour l'extrême gauche sauf, si brutaux et sanguinaires soient-ils, ceux qui ont la bonne idée de se prétendre de gauche, ou à tout le moins anti-impérialistes, à qui il est beaucoup pardonné.

Les Sex Pistols chantaient naguère, avec la conviction confuse de la jeunesse, God Save the Queen and her Fascist Regime. Pour Roland Barthes jadis, soucieux de plaire à la jeunesse soixante-huitarde, " la langue est fasciste ", - aimable enfantillage dont on se souvient un peu, - et pour l'antipsychiatrie des années 1960 qui avait alors le vent en poupe, tout psychiatre était un « fasciste ».

33 À la longue, les conservateurs ont compris le truc et aux États-Unis, ils dénoncent désormais à qui mieux mieux les « liberals », les intellectuels de gauche comme les vrais fascistes. On observe cette manœuvre de rétorsion dans Liberal Fascism de Jonah Goldberg: la gauche US n'a cessé, de Roosevelt à Hillary Clinton, de défendre des principes « remarquablement semblables » à ceux de Hitler et de Mussolini, expose cet ouvrage de ton académique (2007). Au même moment en France, le secrétaire d'État R. Karoutchi dénonce en 2008 les attaques de la presse de gauche contre le Président Nicolas Sarkozy comme l'expression d'un « fascisme rampant ».

Aujourd'hui, Endehors.org s'en prend au "nouveau fascisme » qu'est le féminisme radical. Un site écologiste a repéré d'autres « fascistes » à combattre, ce sont les Global Warming Skeptics, tous ceux qui mettent en doute le réchauffement planétaire - mais www.ecofascism.com rétorque du tac au tac: «Environmentalism is Fascism!» Le catégorème diabolisant, tout usé jusqu'à la corde qu'il soit, continue donc sur sa lancée sur le web.

Un autre activisme ostentatoire anti-, l'« antiracisme », est apparu en rapides progrès dans les années 1980 et a pris à point nommé «la relève d'un antifascisme bien fatigué » (Gauchet $2002: 222$ ). Lui aussi a dûment suivi la pente de l'instrumentalisation tous azimuts et de la déperdition sémantique concomitante. "Racisme » est appliqué désormais à toute discrimination alléguée quelle qu'en soit la nature et s'est regrettablement vidé de tout sens précis.

Quant à "Islamo-fascisme", il s'est répandu de nos jours avec quelques bons arguments comparatifs et un gros potentiel d'anachronisme. Si j'applique sans plus " totalitarisme » ou « fascisme » au régime ba'asiste irakien ou au régime des El-Assad en Syrie, je feins parfois de croire que cet étiquetage permet de faire l'économie d'une comparaison en forme au-delà de circonstances géopolitiques incommensurables. 


\section{Un projet en cours}

37 Je ne compte pas conclure cette esquisse, il n'y a pas lieu de le faire, mais je veux signaler que mon travail actuel s'inscrit dans cette problématique de la rhétorique de la qualification et des controverses d'étiquetage.

J'ai publié naguère une monographie, L'immunité de la France envers le fascisme : un demisiècle de polémiques historiennes. Je cherchais à y reconstituer une controverse académique de longue durée et à l'interpréter dans son contexte historique. La polémique sur laquelle j'ai travaillé, polémique franco-française et internationale étendue sur un demi-siècle, 1954-2005, porte sur l'existence en France au $20^{\mathrm{e}}$ siècle de quoi que ce soit, - doctrines, programmes, mouvements, événements, régime - que l'on puisse rapporter au "fascisme ». Elle est ponctuée de débats acerbes et d'attaques violentes, celles notamment qui ont accompagné en France les livres successifs de l'Israélien Zeev Sternhell.

39 J'ai élargi mon enquête dans une étude parue en 2013 qui prend cette fois à bras le corps les débats entre historiens occidentaux depuis 1945 sur le "Fascisme générique " et le fascisme en tel et tel pays ${ }^{14}$. " Fascisme » forme un nœud gordien de définitions et de typologies incompossibles - et ce, bien avant que les historiens académiques ne s'en emparent: depuis 1922, depuis que les popolari, les socialistes et les communistes italiens en exil ont commencé à se déchirer sur le sens et la nature du phénomène qui les chassait de leur pays, depuis les disputes interminables dans le sein du Komintern et avec les trotskystes et oppositionnels jusqu'aux controverses incessantes entre deux générations d'historiens des deux mondes de 1950 à nos jours. La compilation des définitions et interprétations académiques du fascisme a abouti en anglais à cinq gros volumes. Une sous-controverse sans issue, parmi bien d'autres, oppose par exemple les historiens qui, comme Ernst Nolte, ne conçoivent le fascisme que "dans son époque ", issu de la Première Guerre mondiale, de ses massacres et de la réaction à la "menace " communiste, c'est-à-dire les historiens pour qui le fascisme, c'est 1914-1945 sur le seul continent européen, et ceux qui, à l'instar de l'Américain Robert Paxton, du Britannique Roger Griffin pensent qu'il s'agit d'une virtualité persistante dans toutes les sociétés modernes. Il convient à l'historien intellectuel, non de bricoler à son tour, après tout le monde, une définition syncrétique de plus de "fascisme »- en vue de chercher à mettre d'accord les Nolte, Sternhell, Griffin, Paxton, Milza, Gentile de ce monde ! - mais de rendre raison de cette dynamique de mésentente même, de donner à comprendre la « logique » à l'œuvre dans les controverses intellectuelles de cette sorte et d'expliquer leur caractère inépuisable et insurmontable et, à de certains égards, fructueux.

« Fascisme » est un terme catégoriel qui est de part en part historique, à savoir qui n'est déduit ni de l'entendement ni du sens commun, mais qui permet de regrouper un ensemble d'objets - en l'espèce des régimes politiques qui seront donnés pour essentiellement équivalents et co-intelligibles selon des paramètres qui n'ont de sens que dans l'histoire. Autrement dit, aucun arbitrage décisif ne permet de retenir ou d'écarter de façon concluante les raisons avancées pro \& contra. Un historien anglais, Stanley Payne, a comparé les définitions savantes de «fascisme » depuis un demi-siècle, définitions successivement mises à mal par les objections des uns et des autres, à un champ de bataille le soir venu, couvert de carcasses d'armement hors d'usage et abandonnées (1995). 
41 Fascisme, national-socialisme et communisme furent-ils, à titre identique ou du moins essentiellement comparable, des «totalitarismes»? Ici, on rencontre une vaste bibliothèque pour et contre que je parcours au volume II, sous presse, de Fascisme, totalitarisme, religion séculière: trois concepts pour le $20^{e}$ siècle. "Totalitarisme" est le terme le plus disputé du vocabulaire politique et historiographique depuis bientôt un siècle. "Totalitarisme » s'est trouvé approprié non pas depuis la Guerre froide, mais bien depuis les années 1920 par tous les courants politiques et toutes les écoles historiennes - rejeté, répudié, revendiqué, argumenté et contre-argumenté, défini et contre-défini et il a servi à « qualifier » des régimes et à les assimiler - au premier chef, à l'indignation précisément des historiens "antifascistes", le régime bolchevik/ stalinien et le régime nazi.

42 La notion complémentaire, généalogique, de «religions politiques» nouvelles, affrontées dans une lutte à mort, mais ayant les mêmes origines et copiées les unes des autres, invite non moins à confronter. Les idéologies « totales » du siècle passé, qu'elles soient réputées de gauche ou de droite, ont créé des "vies inutiles ", elles ont légitimé le meurtre de misérables par milliers, elles ont conçu et justifié le recours à la terreur en vue de soumettre la société à un remodelage intégral. De quel nom désigner la nature de telles convictions? Ce sont ces sortes de croyances extrêmes, apparemment différentes de nature des autres programmes et projets politiques que, dès 1931, le philosophe catholique Waldemar Gurian a précisément baptisées des «religions totalitaires ». D'innombrables chercheurs, non moins en conflit entre eux, identifient, à la source du malheur du siècle, la "sacralisation de la politique ", - en d'autres termes l'émergence, l'hégémonie et puis la dissolution de politische Religionen, de Political religions, de religions politiques ou séculières. Le $20^{\mathrm{e}}$ siècle aurait été alors une suite de guerres de religion. "Ours has been the age par excellence of political faiths, of secular salvations offered on a national or universal scale» (Koenker. 1965 : VII). Les religions politiques rouge, noire et brune, auraient été, comme les fanatiques religions révélées l'ont été autrefois, responsables des massacres et des crimes qui jonchent le siècle crimes, comme toujours, commis au nom du Souverain bien.

43 À la fin des années 1930 à Vienne, Eric Vœgelin, ce penseur spiritualiste que le monde francophone "découvre " avec un bon demi-siècle de retard, avait un des premiers caractérisé l'essence de la modernité comme tenant à l'apparition et aux progrès de "religions politiques» élevant dans un monde privé de transcendance, ce qu'il nommait un Realissimum, une Idole plus-que-réelle, - l'État, la Production économique, la Science, la Race et le Sang, la Nation, la Classe (1938). Un troisième concept infiniment controversé traverse dès lors la pensée historique du $20^{e}$ siècle, le concept de "religion séculière/religion politique ». Ein Gespenst geht um : il s'agit d'une sorte de spectre conceptuel qui vient hanter plusieurs grandes théories politiques et historiques sans jamais tout à fait prendre corps comme une notion partagée communément par l'ensemble des chercheurs. Mais c'est un concept dont certains penseurs, sans s'accorder en tout, il s'en faut, Norman Cohn, Karl Löwith, Eric Vœgelin, font du moins un puissant instrument herméneutique, un moyen d'apercevoir la dynamique de la modernité sous l'angle d'une interprétation qui ne soit pas auto-justificative.

Le conflit entre les trois catégories elles-mêmes est patent : le concept de «fascisme » [générique] repose sur l'idée que les dictatures ultra-nationalistes qui sévissent en Europe dans les années 1920-40 étaient semblables entre elles par des traits essentiels mais fondamentalement différentes de la dictature bolchevique avec laquelle elles 
étaient en conflit frontal, - le concept de "totalitarisme " suppose au contraire qu'elles étaient semblables ou comparables à plusieurs ou à tous les égards. Il invite à la comparaison (sans que cette comparaison qui, elle aussi, s'amorce dès les années d'avant-guerre, impose le recours à ce terme) entre les régimes soviétique, fasciste et nazi. D'où les hauts cris qui accueillent toujours, dans une partie de la gauche européenne du moins, l'«amalgame " de totalitarisme, confrontant sous divers paramètres - et non assimilant du reste - le scélérat nazisme et le communisme, censément perverti mais plein de bonnes intentions, et ce, quelle que soit la force heuristique de ce concept propre au $20^{\mathrm{e}}$ siècle.

\section{BIBLIOGRAPHY}

Altwegg, Jürg. 1989. Querelles de français [Traduit de l'allemand, Republik des Geistes] (Paris : Grasset)

Amossy, Ruth. 2014. Apologie de la polémique (Paris : PUF)

Angenot, Marc. 2003. Anarchistes et socialistes : trente-cinq ans de dialogue de sourds. (Montréal : Discours social). Étude reprise en grande partie sous le même titre dans l'ouvrage collectif de Jacqueline

Dangel, Gilles Declercq \& Michel Murat (éds). 2003. La Parole polémique (Paris : Champion)

Angenot, Marc. 2008. Dialogues de sourds. Traité de rhétorique antilogique (Paris : Mille et une Nuits)

Angenot, Marc. 2013. Fascisme, totalitarisme, religion séculière : trois concepts pour le XXe siècle. Notes pour un séminaire d'histoire conceptuelle. Volume 1. Catégories, Idéaltypes - Fascisme (Montréal : Discours social)

Angenot, Marc. 2009. L'immunité de la France envers le fascisme : un demi-siècle de polémiques historiennes. Suivi de : Le fascisme dans tous les pays (Montréal : Discours social)

Angenot, Marc. 2005 [1982]. La parole pamphlétaire, contribution à la typologie des discours modernes(Paris : Payot /Rivages)

Angenot, Marc. 2004. Rhétorique de l'anti-socialisme. Essai d'histoire discursive, 1830-1914 (Québec :

Presses de l'Université Laval)

Augstein, Rudolf \& al. 1987. Historikerstreit : die Dokumentation der Kontroverse und die Einzigartigkeit der nationalsozialistischen Judenvernichtung (München : Piper)

Barthes, Roland. 1953. Le degré zéro de l'écriture suivi de Nouveaux essais critiques (Paris : Seuil)

Bödecker, Hans E. 2002. Begriffsgeschichte, Diskursgeschichte, Metapherngeschichte (Göttingen :

Wallstein)

Bruneteau, Bernard. 2010. Le totalitarisme. Origines d'un concept, genèse d'un débat, 1930-1942 (Paris : Cerf)

Conquest, Robert. 1986. Harvest of Sorrow : Soviet Collectivization and the Terror-Famine(New York : Oxford University Press) 
Gauchet, Marcel. 2002. La démocratie contre elle-même (Paris : Gallimard)

Goldberg, Jonah. 2007. Liberal Fascism : The Secret History of the American Left, From Mussolini to the Politics of Change (New York : Doubleday)

Guilhaumou, Jacques. 2006. Discours et événement. L'histoire langagière des concepts (Besançon : Presses Universitaires de Franche-Comté)

Gurian, Waldemar. 1931. Der Bolschewismus : Einführung in Geschichte und Lehre(Freiburg i. B. : Herder)

Gurian, Waldemar. 1935. Bolschewismus als Weltgefahr (Luzern : Vita nova)

Hagège, Claude. 2000. Halte à la mort des langues (Paris : Odile Jacob)

Koenker, Ernest Benjamin. 1965. Secular Salvations: The Rites and Symbols of Political Religions (Philadelphia PA : Fortress Press)

Koselleck, Reinhart. 2002. The Practice of Conceptual History : Timing History, Spacing Concepts. Foreword by Hayden White (Stanford : Stanford University Press) [Recueil de divers essais théoriques traduits en anglais]

Koselleck, Reinhart (éd.). 1979. Historische Semantik und Begriffsgeschichte (Stuttgart : Klett-Cotta)

Lemkin, Raphael. 1944. Axis Rule in Occupied Europe : Laws of Occupation, Analysis of Government, Proposals for Redress (Washington DC : Carnegie Endowment for International Peace, Division of International Law)

Machefer, Philippe. 1974. Ligues et fascismes en France, 1919-1939 (Paris : PUF)

Micheli, Raphaël. 2013. «Les querelles de mots dans le discours politique : modèle d'analyse et étude de cas à partir d'une polémique sur le mot "rigueur" ", Argumentation et Analyse du Discours 10, [en ligne : http://aad.revues.org/1446, consulté le 20 septembre 2014]

Moirand, Sophie et Rémy Porquier. 2008. « De l'éthique de la nomination à l'éthique de l'interprétation : autour du mot "otage" et de quelques autres ", Régine Delamotte-Legrand \& Claude Caitucoli (éds.), Morales langagières. Autour de propositions de recherche de Bernard Gardin (Rouen : Presses des Universités de Rouen et du Havre), 139-154

Naimark, Norman. 2010. Stalin's Genocides (Princeton : Princeton UP)

Nolte, Ernst. 1963.Der Faschismus in seiner Epoche. Action francaise - Italienischer Faschismus Nationalsozialismus (München : Piper)

Ory, Pascal. 1995. La France allemande. Édition revue (Paris : Gallimard, Folio Hist.)

Payne, Stanley. 1995. A History of Fascism, 1914-1945 (London : UCL)

Pétré-Grenouilleau, Olivier. 2005. Traites négrières, essai d'histoire globale (Paris : Gallimard)

Rémond, René. 2006. Quand l'Etat se mêle de l'histoire. Entretiens avec François Azouvi (Paris : Stock, Les essais)

Richter, Melvin. 1995. The History of Political and Social Concepts : A Critical Introduction (New-York \& Oxford : Oxford University Press)

Taguieff, Pierre-André.1990. « L'argumentation politique. Analyse du discours et Nouvelle rhétorique. A la mémoire de Chaïm Perelman, 1912-1984 », Hermès 8-9, 261- 286

Taguieff, Pierre-André. 2008. Le nouveau national-populisme (Paris : CNRS Éditions)

Taguieff. 2007. Les contre-réactionnaires. Le progressisme entre illusion et imposture (Paris : Denoël) 
Voegelin, Erich. 1994. Die politischen Religionen (Wien : Bermann-Fischer, 1938) [En français, traduit avec 58 ans de retard, Les religions politiques (Paris : Cerf)

\section{NOTES}

1. Voir aussi parmi de nombreux autres ouvrages sur la Querelle des historiens allemands, Augstein \& al., 1987.

2. La Begriffsgeschichte a essentiellement travaillé les « kulturphilosophischen Grundbegriffe », les mots-concepts fondamentaux de la vie civique et politique ; elle les a étudiés souvent sur la durée de plusieurs siècles en remontant à la Renaissance, au moyen âge : ce sont Liberté, Égalité, Fraternité somme toute et autres "grands mots" pareils. Voir à titre d'introduction le collectif sous la direction de Hans E. Bödecker, 2002.

3. Site de France TVInfo, 510 2013. Quant à Laurent Joffrin, directeur du Nouvel Observateur, il se dit prêt à aller devant les tribunaux pour confirmer le droit de qualifier comme il l'entend : «Marine Le Pen ne veut pas qu'on qualifie le Front national de parti "d'extrême droite". Certes. Malheureusement, nous ne voyons pas comment le qualifier autrement. Qu'y a-t-il à droite du Front national ? Quelques groupuscules marginaux qui ne jouent aucun rôle sur la scène électorale. Dès lors le Front national se situe, de toute évidence, sur le bord extrême du spectre politique, et bien sûr sur la droite et non sur la gauche. C'est l'UMP qui est à droite. Le FN est bien d'extrême-droite, comme l'atteste par ailleurs son programme ». 17. 10. 2013 (en ligne).

4. «Le FN est-il ou pas un parti d'extrême droite ? Non seulement c'est un parti d'extrême droite mais c'est même plus que ça, c'est un parti national-fasciste» (Libération, 8 oct. 2013).

5. Ou bien dois-je proclamer par principe «l'unicité» de la Shoah, comme font Alain Besançon et plusieurs autres penseurs? Mais Besançon le fait avec des arguments mystiques qui n'entrent pas en dialogue avec les victimes non-eurocentriques de massacres de masse.

6. Au premier chef les Tasmaniens - un des génocides intégralement accomplis de l'histoire coloniale, sur lequel il a été à l'époque beaucoup écrit (mais rien de réédité). En 1873, Truganini, la dernière survivante de ces peuples aborigènes, est emmenée à Hobart. Elle y décède en 1876.

7. Suivi du «nettoyage ethnique » de 30.000 réfugiés dans la région.

8. La totalité des populations tchétchène et ingouche - un demi-million d'hommes, femmes et enfants - a été déportée en 1944 en quelques jours en Kazakhie et Kirghizie.

9. Il ajoute : "This argument is made more difficult by the fact that there was no single act of genocide in the Soviet case, but rather a series of interrelated attacks on "Class enemies" and "enemies of the people", metonyms for diverse alleged opponents of the Soviet state. "

10. http://leprofesseurmasque.blogspot.ca/2012/02/les-abus-de-langage.html

11. Le président du collectif des Antillais Guyanais-Réunionnais, Patrick Karam, devait annoncer le 3 février 2006, le retrait de la plainte.

12. Dans son ouvrage, l'historien tentait d'éclairer avec objectivité et équanimité les différentes formes d'esclavage qui ont sévi au cours des siècles en Afrique : africaine, arabe (dans l'Océan Indien) et occidentale. En ne se limitant pas à la « dénonciation » des seuls Européens, il choquait aussi certains de ses dénonciateurs, notamment les Africains de confession musulmane.

13. www.lph-asso.fr/index.php?option=com_content\&view=article\&id=2\&Itemid=13\&lang=fr On trouve dans cette pétition (en ligne) des distinguos qui me semble importants à retenir : «L'histoire n'est pas la morale. L'historien n'a pas pour rôle d'exalter ou de condamner, il explique. L'histoire n'est pas l'esclave de l'actualité. L'historien ne plaque pas sur le passé des schémas idéologiques contemporains et n'introduit pas dans les événements d'autrefois la sensibilité d'aujourd'hui. L'histoire n'est pas la mémoire. L'historien, dans une démarche scientifique, recueille les souvenirs des hommes, les compare entre eux, les confronte aux documents, aux objets, aux traces, et établit les faits. L'histoire tient compte de la mémoire, elle 
ne s'y réduit pas. L'histoire n'est pas un objet juridique. Dans un Etat libre, il n'appartient ni au Parlement ni à l'autorité judiciaire de définir la vérité historique. La politique de l'Etat, même animée des meilleures intentions, n'est pas la politique de l'histoire. »

14. Le volume 4 comportera des annexes : «Religion, sacré, dogme, croyance» et «Religion civile».

\section{ABSTRACTS}

This paper deals with a specific subcategory in eristic rhetoric and the polemic, i.e. the bitter and oftentimes endless disputes regarding name tags and labelling in the political realm and the public life. It aims at extending the concept of qualification beyond the legal realm in dealing with the contentious issue of categorizing and naming, and shows what appears to be at stake in some typical cases. It then focuses on two issues: the disagreements between historians regarding the use in historiography of some legal categories such as "genocide", and the techniques of demonization, more specifically the use and abuse over the last half-century of name tags such as "fascist", "totalitarian" and the like.

Cet article traite d'un cas d'espèce dans le vaste domaine de la rhétorique éristique et la polémique, à savoir les amères et interminables controverses qui ne portent que sur la qualification - au sens juridique - d'un événement ou d'un individu, d'une entité politique. Il étend donc cette notion de droit pour aborder la question générale des controverses d'étiquetage dont il montre la récurrence quotidienne. Il s'arrête notamment à un exemple prégnant de controverses de qualification juridique utilisées par les historiens avec la catégorie de " génocide » et ses abus. Il aborde l'étiquetage accusateur et « diabolisant » et ses catégories bien connues en longue durée comme « fasciste », « totalitarisme » etc.

\section{INDEX}

Mots-clés: diabolisation, étiquetage, fasciste, génocide, polémique, qualification juridique

Keywords: demonization, fascist, genocide, labelling, legal qualification, polemic

\section{AUTHOR}

\section{MARC ANGENOT}

Université McGill 